\title{
Antoine Bernard De Raymond - En toute saison : le marché des fruits et légumes en France
}

Rennes, Presses universitaires de Rennes ; Tours, Presses universitaires François-Rabelais de Tours, 2013, 306 pages

\section{Pierre Naves}

\section{(2) OpenEdition}

\section{Journals}

Electronic version

URL: http://journals.openedition.org/economierurale/4527

DOI: 10.4000/economierurale.4527

ISSN: 2105-2581

\section{Publisher}

Société Française d'Économie Rurale (SFER)

\section{Printed version}

Date of publication: 20 December 2014

Number of pages: 85-86

ISSN: 0013-0559

\section{Electronic reference}

Pierre Naves, «Antoine Bernard De Raymond - En toute saison : le marché des fruits et légumes en France », Économie rurale [En ligne], 344 | novembre-décembre 2014, mis en ligne le 01 janvier 2014, consulté le 23 septembre 2020. URL : http://journals.openedition.org/economierurale/4527 DOI : https://doi.org/10.4000/economierurale.4527 
Antoine BERNARD DE RAYMOND

\section{En toute saison : le marché des fruits et légumes en France}

Rennes, Presses universitaires de Rennes : Tours, Presses universitaires François-Rabelais de Tours, 2013, 306 p.

D ans un contexte de renouveau des circuits courts de proximité et de remise en cause de I'hégémonie de la grande distribution sur le commerce des fruits et légumes, ce livre offre une analyse détaillée de l'ensemble des transformations ayant influencé le commerce de ces produits en France depuis la fin du dix-neuvième siècle. Antoine Bernard de Raymond est sociologue à I'INRA dans l'unité RITME (Risques, Travail, Marchés, État) et a soutenu en 2007 sa thèse "Maximes empiriques de l'activité économique : le marché des fruits et légumes en France (1936-2006) " à I'ENS Cachan. Tiré largement de cette thèse, le présent ouvrage propose une sociologie de la genèse d'un marché national des fruits et légumes marqué par une path-dependency par rapport aux politiques mises en place au niveau national et européen, aux stratégies des secteurs amont et aval de la filière et qui aujourd'hui pèsent encore sur son fonctionnement. L'auteur s'appuie pour étayer son propos sur l'examen d'un siècle de réformes réglementaires depuis celle du marché des halles parisien en 1896 à celle de l'Organisation commune de marché (OCM) Fruits et Légumes en 1996, des données économiques, ainsi que des entretiens avec des producteurs, des consultants, des distributeurs, etc.

Présenter cet ouvrage de manière chronologique n'est pas chose aisée de par son architecture, ce qui nous amènera à dégager des axes de lecture qui, nous l'espérons, ne trahiront pas la pensée de Bernard de Raymond. Le premier axe renvoie à la genèse du marché national (chapitres 1, 2 et 4 ), le second à la question de la définition des produits et des systèmes visant à les garantir (chapitre 1, 6,7 et 8 ). Nous reviendrons dans un dernier temps sur la question centrale qui anime cet ouvrage, celle de la dématérialisation du marché qui est, in fine, celle du passage d'une logique de commerce à une logique de distribution (chapitres 3 , 4, 5 et 9 ).

Partant de l'examen de la réforme de 1896 visant à régler la question de la représentation des producteurs de province et celle du statut des intermédiaires sur le marché des halles centrales à Paris (dont la spécificité est d'être pensé comme un marché local et national), l'auteur montre comment elle a entériné la centralisation du marché national des fruits et légumes autour de la capitale, tout en cherchant à protéger les producteurs provinciaux de l'opportunisme de certaines catégories d'intermédiaires. La création d'un véritable espace marchand national n'aura lieu qu'à partir des années 1950, avec la création d'un réseau de marchés modernes, les Marchés d'intérêt national (MIN) censés garantir la transparence des échanges grâce à la fixation d'un cours national. Au cours de la période 1965-1970, on assiste à un tournant dans la politique des MIN avec le passage d'une logique de clarification des transactions à une logique de concentration et d'industrialisation de la filière, pour répondre à la concentration du secteur aval. Les MIN, dans ce contexte, apparaissent comme " des espaces de segmentation du marché et de protection des pans traditionnels de la filière » (p. 150) concurrencés par l'émergence des grossistes-livreurs dont les prestations de service, reposant sur la vente à distance, renforcent la dématérialisation du commerce en fruits et légumes et menacent la position des grossistes de carreau dont l'expertise est fondée sur leur capacité à gérer l'incertitude en situation. 
La dématérialisation croissante des échanges permise par le recours aux nouvelles technologies de l'information impose à l'ensemble des acteurs de la filière de nouvelles conventions de qualité. L'enjeu réside premièrement dans la redéfinition du statut de certains produits (agrumes) pour en faire des produits de consommation courante, alors qu'ils étaient jusque-là des produits réservés à certaines catégories sociales. Deuxièmement, l'édiction de normes de commercialisation dans le cadre de l'OCM Fruits et Légumes prévue par le traité de Rome en 1957 permet de standardiser et normaliser la qualité des produits, celleci devant pouvoir être mesurée en fonction de critères objectivables afin de permettre la généralisation du commerce à distance. Enfin, le recours aux techniques de production modernes permet dans une certaine mesure aux producteurs de $s$ affranchir des contraintes saisonnières de production pour approvisionner le marché toute l'année. La conjugaison de ces trois éléments (redéfinition du statut des produits, normalisation de la qualité et " désaisonnalisation " du marché) ouvre la voie au passage d'une logique de commerce à celle de la distribution.

Alors qu'ils avaient vocation à organiser un réseau de distribution moderne à l'échelle nationale, très vite les MIN se trouvent dépassés par les évolutions du commerce de gros, dont les acteurs sont désormais potentiellement situés en dehors de leur espace physique. En effet, la grande distribution naissante tend à les court-circuiter en créant des centrales d'achats et s'impose rapidement comme le débouché principal de la filière. Face à l'atomicité de l'offre, elle se trouve en mesure d'imposer ses règles du jeu à l'ensemble de la filière, dont les tentatives d'organisation restent de peu d'effets face à la concentration du secteur aval. L'exemple des référentiels privés mis en place par la grande distribution et dont l'obtention conditionne l'accès aux rayons des magasins est en ce sens tout à fait révélateur de sa montée en puissance dans la gouvernance de la filière. Bernard de Raymond montre ainsi que ces standards censés garantir des procédés culturaux respectueux de l'environnement permettent d'asseoir la diffusion de bonnes pratiques gestionnaires au sein de l'exploitation, accréditant l'idée d'un " tournant managérial de l'agriculture " imposé par le secteur aval.

Cet ouvrage, par la richesse et le détail de ses analyses, offre donc un point de vue stimulant et original sur le marché français des fruits et légumes. II montre comment ce marché s'est constitué et quels furent les acteurs qui ont contribué à lui donner sa forme actuelle (désaisonnalisé et standardisé). Ce livre donne surtout à voir une redéfinition des rapports de force au sein de la filière, de plus en plus contrôlée par un aval qui parvient à imposer ses propres normes et critères de qualité à l'ensemble des autres acteurs. Pour autant, l'auteur prend le parti de n'étudier qu'une partie de la filière fruits et légumes, la filière " organisée » et construite sur le mode coopératif, ce qui l'amène à passer sous silence la variabilité des modes de commercialisation des fruits et légumes et leur impact sur la répartition de la valeur ajoutée le long de la filière, ainsi que les stratégies des collectivités territoriales visant à réinscrire les modes de distribution dans un contexte de proximité géographique et relationnelle. Nonobstant ces angles morts qui sont autant d'invitations à effectuer de nouvelles recherches sur ce secteur et les logiques territoriales qui le traversent, l'intérêt de ce livre est d'attirer l'attention sur les processus ayant entraîné la déterritorialisation et la désaisonnalisation de l'agriculture et des modes de consommation, et nous invite à penser le marché de manière globale, comme une construction sociale, économique, politique et technique.

Pierre NAVES

Doctorant

Centre Émile Durkheim (UMR-5116) Université Bordeaux Segalen 